\title{
Novel techniques for the analysis of the TOA radiometric uncertainty
}

\author{
Javier Gorroño*a, b, Andrew Banks ${ }^{\mathrm{a}}$, Ferran Gascon ${ }^{\mathrm{c}}$, Nigel P. Fox ${ }^{\mathrm{a}}$, Craig I. Underwood ${ }^{\mathrm{b}}$ \\ ${ }^{a}$ National Physical Laboratory, NPL, Hampton Road, Teddington, Middlesex TW11 OLW, UK; \\ bSurrey Space Centre, University of Surrey, Guildford, Surrey, GU2 7XH, UK; \\ ${ }^{c}$ European Space Agency, ESA/ESRIN, Via Galileo Galilei, 00044 Frascati, Italy
}

\begin{abstract}
In the framework of the European Copernicus programme, the European Space Agency (ESA) has launched the Sentinel-2 (S2) Earth Observation (EO) mission which provides optical high spatial -resolution imagery over land and coastal areas. As part of this mission, a tool (named S2-RUT, from Sentinel-2 Radiometric Uncertainty Tool) estimates the radiometric uncertainties associated to each pixel using as input the top-of-atmosphere (TOA) reflectance factor images provided by ESA. The initial version of the tool has been implemented — code and user guide available ${ }^{1}$ — and integrated as part of the Sentinel Toolbox. The tool required the study of several radiometric uncertainty sources as well as the calculation and validation of the combined standard uncertainty in order to estimate the TOA reflectance factor uncertainty per pixel. Here we describe the recent research in order to accommodate novel uncertainty contributions to the TOA reflectance uncertainty estimates in future versions of the tool. The two contributions that we explore are the radiometric impact of the spectral knowledge and the uncertainty propagation of the resampling associated to the orthorectification process. The former is produced by the uncertainty associated to the spectral calibration as well as the spectral variations across the instrument focal plane and the instrument degradation. The latter results of the focal plane image propagation into the provided orthoimage. The uncertainty propagation depends on the radiance levels on the pixel neighbourhood and the pixel correlation in the temporal and spatial dimensions. Special effort has been made studying non-stable scenarios and the comparison with different interpolation methods.
\end{abstract}

Keywords: Sentinel-2, radiometric uncertainty, spectral response, orthorectification

\section{INTRODUCTION}

In the framework of the European Union Copernicus programme, the European Space Agency (ESA) has launched the Sentinel-2 (S2) Earth Observation (EO) mission. S2 includes as main payload the MultiSpectral Instrument (MSI) which consists of 13 Visible and Near-InfraRed (VNIR) and Short-Wave InfraRed (SWIR) bands with spatial resolutions of 10 $\mathrm{m}, 20 \mathrm{~m}$ and $60 \mathrm{~m}$ as well as a short revisit time (5 days at the equator with two satellites) and a wide field of view (290 $\mathrm{km})^{2}$.

As part of this mission, the first version of the S2-RUT (Sentinel-2 Radiometric Uncertainty Tool) has been made available to the community ${ }^{1}$. This tool estimates the radiometric uncertainty associated to each pixel using as input the Top-Of-Atmosphere (TOA) reflectance images provided by ESA. The identified uncertainty contributors are combined following the Guide to the Expression of Uncertainty in Measurement (GUM) in order to provide an estimated uncertainty ${ }^{3}$. This combination model is further validated by comparing the results to a multivariate Monte Carlo Method $(\mathrm{MCM})^{4}$. In addition, it has been studied the correlation among the different uncertainty contributions and the impact of simplifications in the combination model.

The tool software design looks for an efficient strategy to implement the uncertainty estimates. The first version does account for the most important uncertainty contributors and effectively asses their level by modelling the uncertainty levels from the metadata, external sources etc. The tool permits, for example, the assessment of the uncertainty at a desired coverage probability and the selection/deselection of the uncertainty contributors for sensitivity studies. It provides "uncertainty images" coded in a specified user format that include pixel geolocation. The Figure 1 shows an example of the S2-RUT results in the Sentinels Toolbox. The image represents the Albufera Lake in Valencia (Spain) at the 12th of January 2016. The uncertainty — right side of the figure - ranges from $>10 \%$ for open sea to $5-6 \%$ in the lake body, $5-15 \%$ in the rice fields covered by water and $2-4 \%$ in the land areas. 


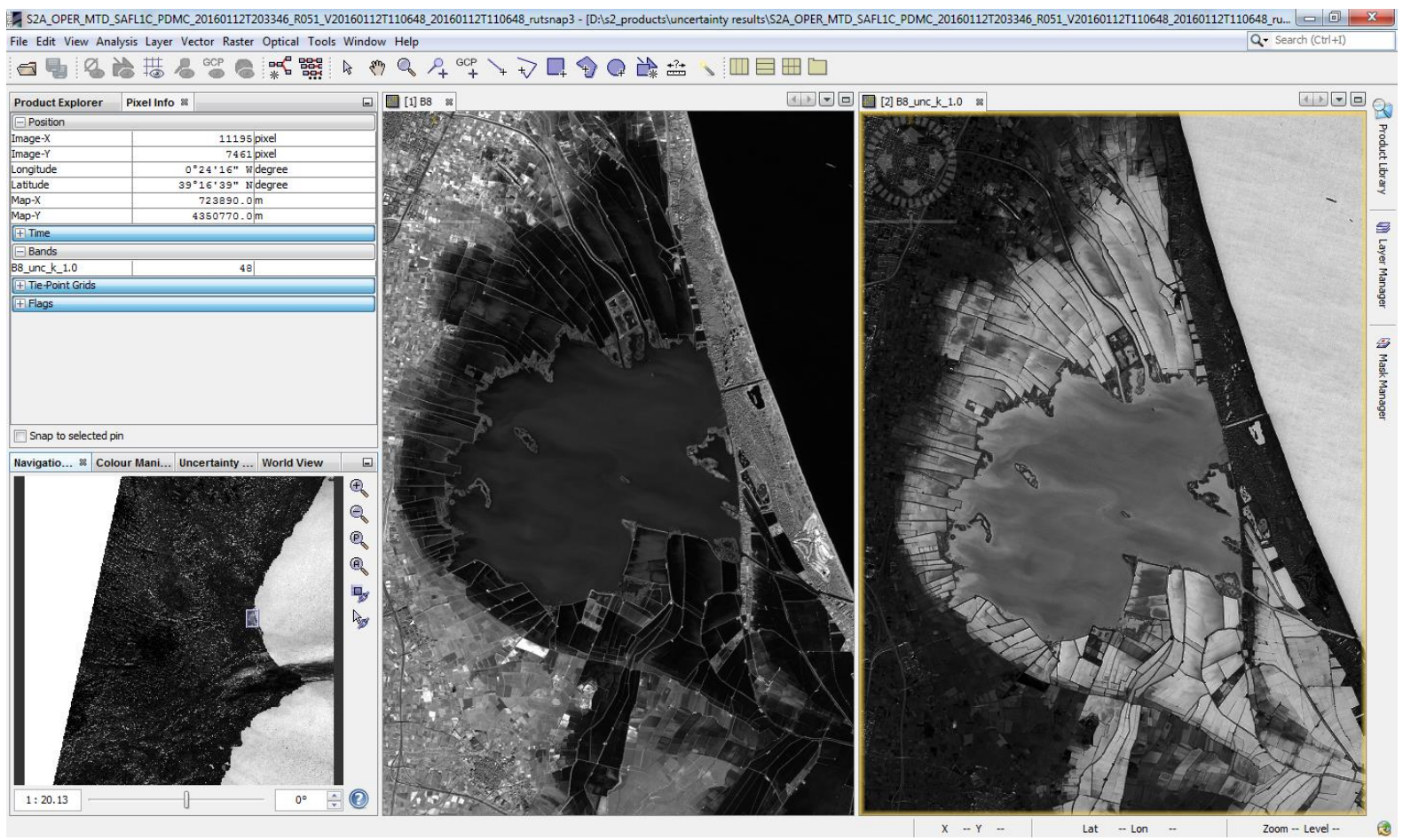

Figure 1 Screen-shot of the Sentinels Toolbox. It contains the pixel info and navigation panels (left), the L1C S2 B8 image for the Albufera lake (centre) and the equivalent uncertainty $k=1$ (right).

Here we describe the recent research in order to accommodate novel uncertainty contributions to the TOA reflectance uncertainty estimates in future versions of the tool. The two contributions that we explore are the radiometric impact of the spectral knowledge in Chapter 2 and the uncertainty propagation of the resampling associated to the orthorectification process in Chapter 3.

\section{SPECTRAL KNOWLEDGE UNCERTAINTY}

The MSI instrument is intended to measure the radiance of a specific region of the spectrum. The effect of the several components along the optical path — telescope, splitter, filter and detectors — determines the final Spectral Response Function (SRF) that is measured at each band. The "equivalent radiance" $L_{\mathrm{eq}}-$ and the converted reflectance factor is defined by the spectral convolution of the scene TOA radiance $L_{\mathrm{TOA}}$ with the instrument SRF:

$$
L_{\mathrm{eq}}(b)=\frac{\int_{\lambda} L_{\mathrm{TOA}}(\lambda) \operatorname{SRF}_{\text {ref }}(\lambda) d \lambda}{\int_{\lambda} \operatorname{SRF}_{\mathrm{ref}}(\lambda) d \lambda}
$$

$\mathrm{SRF}_{\text {ref }}$ here is the reference $\mathrm{SRF}$ that associates the measurements for each band $b$. However, $\mathrm{SRF}_{\text {ref }}$ does not represent the actual SRF of each pixel in the focal plane, at any point in time and with a perfect knowledge. That is, it has an associate uncertainty due to effects of 1) spectral non-uniformity, 2) spectral on-orbit variations and 3) spectral calibration uncertainty. The uncertainty associated to this $\mathrm{SRF}_{\text {ref }}$ translates into a radiometric uncertainty on $L_{\mathrm{eq}}$. Note that here the $\mathrm{SRF}_{\text {ref }}$ is associated to the mean of the pixel's SRF. If each pixel had an associated SRF, the effect of spectral non-uniformity would be suppressed at the expense of an enormous increase in the processing requirements (e.g. atmospheric correction).

Previous work describes an analytical process to determine the uncertainty of the SRF at any interpolated point including the covariance between the measured point ${ }^{5}$. For the study here, the analytical process is more complicated since it is necessary to propagate the SRF uncertainty through the spectral convolution. The TOA spectral radiance $L_{\mathrm{TOA}}$ cannot be easily described as a function that resolves the function analytically. It could be possible to investigate the problem by 
applying a "hybrid" approach that calculates the SRF uncertainty at any interpolated point analytically and propagates the uncertainty through the integral using the MCM approach. The limitation here exist if the algorithm wants to be regularly updated. For example, by using a different interpolation method or changing its weights. In that case a full MCM approach seems a "flexible" solution that will be beneficial if consequent revisions of the code are performed.

The intention here is to introduce an implementation that permits to assess both the radiometric dispersion as a consequence of the spectral knowledge calibration and non-uniformity and the error between the mean of that dispersion and the radiance obtained using the theoretical $S R F_{\text {ref. Figure }} 2$ describes the steps implemented in this first implementation of the contribution:

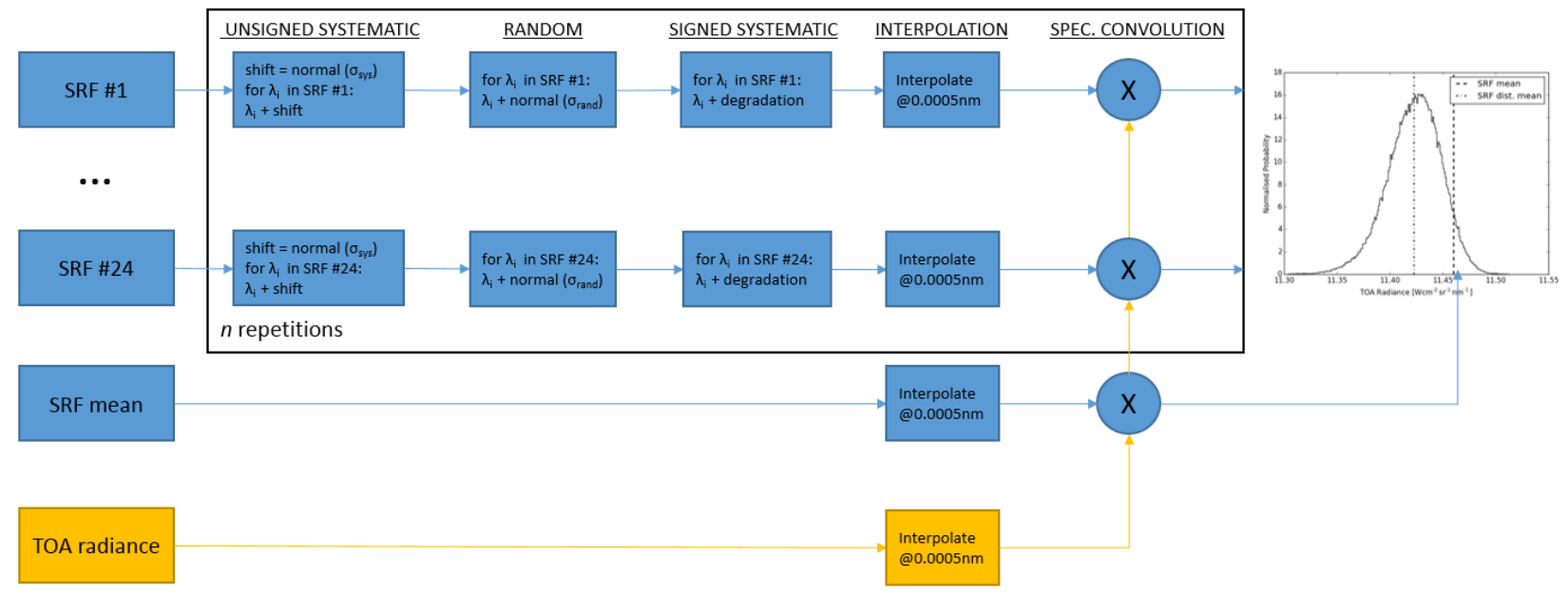

Figure 2. Diagram describing the S2 spectral uncertainty assessment.

The module sets as inputs the SRF measured at instrument level for each VNIR band and a simulated TOA radiance covering the same VNIR range. There are a total of 24 SRFs at $1 \mathrm{~nm}$ spectral resolution that provide an estimation of the spectral non-uniformity effect across the focal plane. There is, nonetheless, a certain limitation since not all the pixels in the focal plane have been measured but only 2 SRFs are available per detector module. Pre-flight as well as post-launch verification have shown how the spectral non-uniformity is mainly produced at the edges of the detectors due to the "etching" effects ${ }^{6}$. A more detailed effect of the spectral non-uniformity could be performed if rather than the SRF at an instrument level, a SRF at component level is used as an input. However, this approach has been discarded since, among other limitations, at a focal plane level, the tele-centricity effect - variations of the optical path of $1-2^{\circ}$ across the focal plane - is not accounted. The TOA spectral radiance has been calculated using MODTRAN 5®. This version includes a minimum spectral resolution of $1 \mathrm{~cm}^{-1}$ — spectral sampling $<0.01 \mathrm{~nm}$ in the VNIR region — which uses a fine description of molecular gases and scattering processes ${ }^{7}$. The model used for the example here simulate conditions in a desert area with a mid-latitude summer atmospheric model and a desert default albedo. Figure 3 illustrates the TOA spectral radiance with the S2 VNIR bands.

Figure 2 also introduces the MCM process (black rectangle) that is further subdivided in the different components affecting the distribution of radiance values. The spectral non-uniformity is accounted as part of the systematic budget but in the process also the spectral calibration knowledge as well as degradation effects must be modelled.

The spectral calibration knowledge is limited by the pre-flight accuracy and precision of the measurements. These have different sources as the source short-term stability, the monochromator calibration, the setup alignment etc. The first implementation of the module divides the effects into random and systematic ones. The implementation of systematic effects associates a sample out of a normal distribution for the entire band measurements whereas the random effect associates a different sample out of a normal distribution for each $1 \mathrm{~nm}$ measurement. Preliminary values of $0.2 \mathrm{~nm}$ standard deviation (SD) for the systematic effect and $0.1 \mathrm{~nm}$ for the random distribution have been provided. 

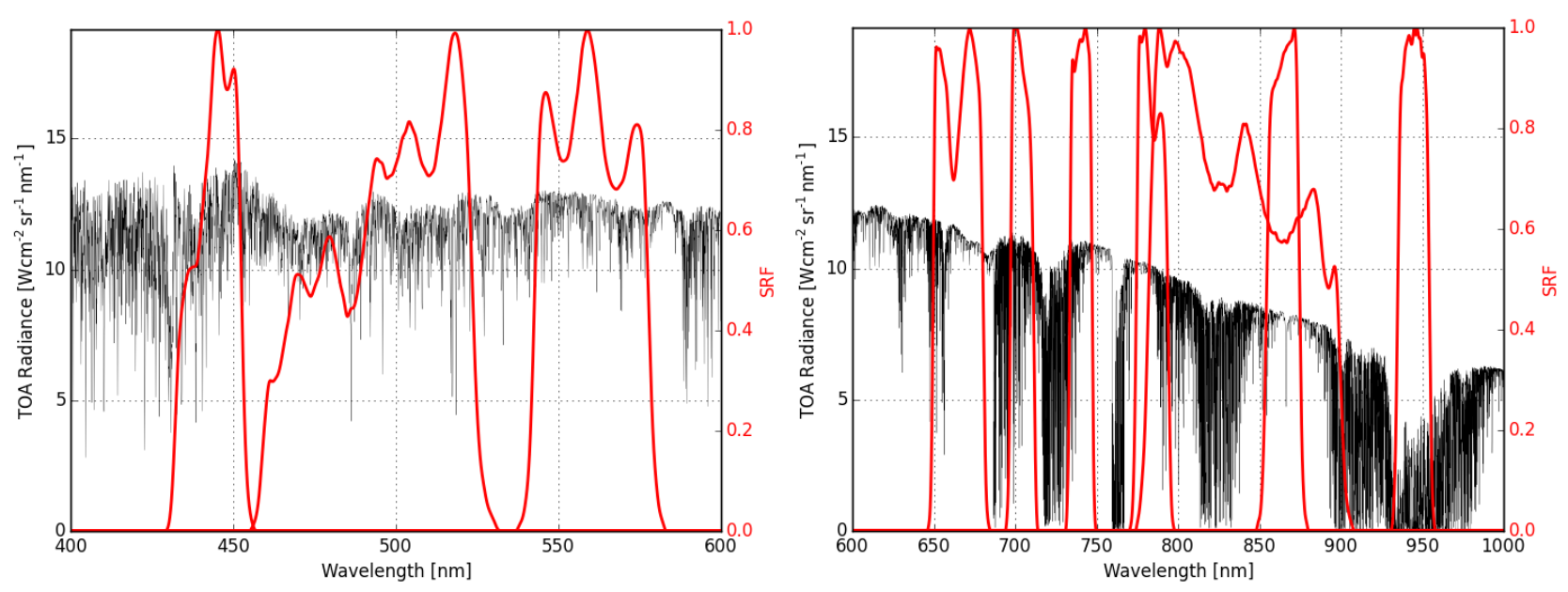

Figure 3. Simulated TOA spectral radiance for a desert case and the S2 bands in the VNIR range.

Figure 2 includes a potential effect of signed systematic errors. They refer to potential variations of the SRF during the mission lifetime. Those variations are mainly produced by temperature variations and the instrument degradation. The type of filters in the S2 MSI - ion-assisted deposition interference filters - tend to have temperature wavelength shift coefficient in the range of $0.01 \mathrm{~nm} / \mathrm{K}^{8,9}$. Thus, the thermal variations are not expected to be a major contribution. However, the degradation could introduce an important spectral variation and needs to be accounted for. A simplified approach has used here a central wavelength shift for each band. The preliminary values used are the degradation of Terra MODIS bands for the first year in-orbit ${ }^{8}$. The closest bands to each Sentinel-2 VNIR band are associated: 0.33(B1), -0.26(B2), 0.04(B3), -0.03(B4), -0.05(B5), -0.07(B6), 0.1(B7), 0.2(B8),-0.18(B8A). These values will be used as an example but, nonetheless, a more detailed study of the potential degradation should follow and, if possible the degradation should specify the in-band variations for bands in the lower wavelength region ${ }^{8}$.

The interpolation of both the TOA radiance and the SRF are performed at $0.0005 \mathrm{~nm}$ steps. This permits sufficient resolution so that small displacements of the wavelength range are well captured. The first interpolation method will use the well-known cubic-spline interpolation. For this interpolation, the values between partition points - knots - are represented by a polynomial of third degree and the first and second derivatives of the interpolation function are continuous in all the range ${ }^{10}$. The Piecewise Cubic Hermite Interpolating Polynomials (PCHIP) method is used to crosscompare and verify results. This interpolation method uses Hermite interpolation conditions that define function values and derivatives are specified at each nodal point. This method provide major flexibility at the cost of some problems in the continuity of the second and higher-order derivatives ${ }^{11}$. Lagrange interpolation or the Barycentric Lagrange interpolation cannot be directly applied here due to the high variation of both the TOA radiance and the SRF ${ }^{12}$. The interference filters introduce several harmonics in the response and does produce a non-convergent interpolation. Here, the Barycentric Lagrange polynomial has been reassessed at each $1 \mathrm{~nm}$ interval with a sufficiently large number of points. This avoids the so-called Runge phenomenon that does typically appear at the edges of the interpolation knots and produces an "apparently" convergent interpolation.

Figure 4 show the results for the previously mentioned interpolation methods and the S2 B1 as example. Interestingly, the Barycentric Lagrange method (as implemented here) tends to approach the cubic spline interpolation. There is a slight difference between the PCHIP and both the cubic spline interpolation and Barycentric Lagrange method with order 9. This situation can be seen in Figure 4 for the wavelengths $437-439 \mathrm{~nm}$ and in between 449 and $451 \mathrm{~nm}$. As commented above, this is the consequence that the former does not require a continuity of the second derivative whereas the latter does. Thus, the results indicate that the S2 interference filters are better interpolated by the PCHIP method due to the less constraining conditions. 

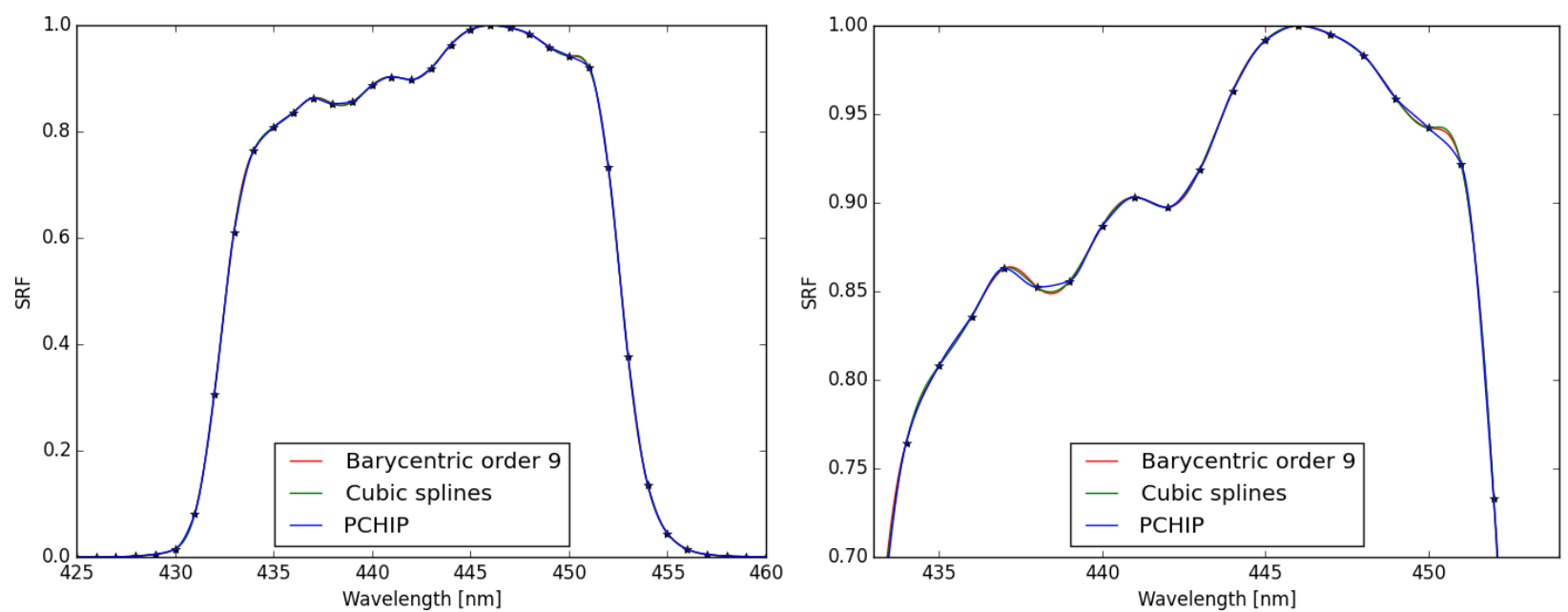

Figure 4. (Left)S2 B1 interpolated SRF and (right) zoom image using Barycentric Lagrange (9th order), cubic splines and PCHIP interpolation methods.

The distribution of the convolved TOA radiance and associated parameters for the Sentinel-2 VNIR bands are given in Figure 5 and Table 1 using the cubic spline interpolation. The same results using the PCHIP interpolation are shown in Figure 6 and Table 2. The Barycentric Lagrange interpolation method as described in Figure 4 has not been integrated in this first iteration of the module. The reason is that, with the method as defined above, the required processing time considerably increases. The B9 has not been included since it is traditionally used for cloud masking. Apart from the traditionally quoted mean, standard deviation (SD) and median, here two more specific parameters will be introduced to better describe the shape of the radiance distribution and provide a better understanding of the instrument and scene properties $^{13}$.

The skewness is a measure of asymmetry and the kurtosis as an indication of the "peakness" of the distribution. A positive skewness value, indicates a distribution tail on the right and vice versa when it is negative. The "excess kurtosis" is calculated by subtracting three to the standard definition of the kurtosis. An "excess kurtosis" with positive values indicate a distribution more peaked than the normal whereas the negative values indicate a distribution less peaked than a normal one. The definition of these two variables is ${ }^{14}$ :

$$
\text { skewness }=\frac{m_{3}}{m_{2}^{3 / 2}} ; \text { kurtosis }=\frac{m_{4}}{m_{2}^{2}}
$$

Where the term $m_{r}$ is associated to the $r$ th moment of the distribution defined for $n$ observations as:

$$
m_{\mathrm{r}}=\frac{1}{n} \sum_{i=1}^{n}\left(x_{i}-\bar{x}\right)^{r}
$$

The Table 1 and Table 2 also include the equivalent radiance $L_{\mathrm{eq}}(b)$ as described in (1) and the error with respect to the mean of the resulting distribution. 

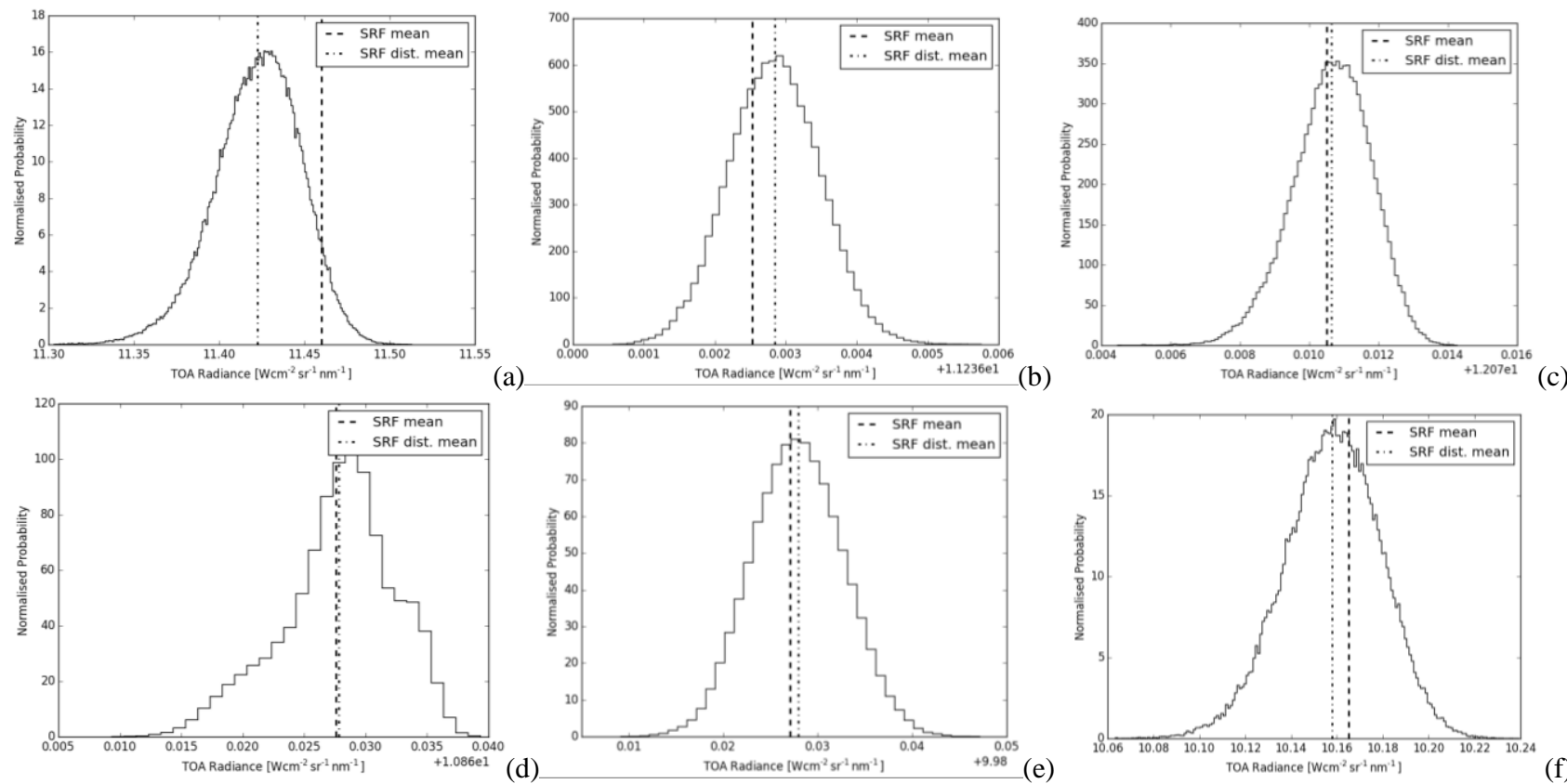

(c)
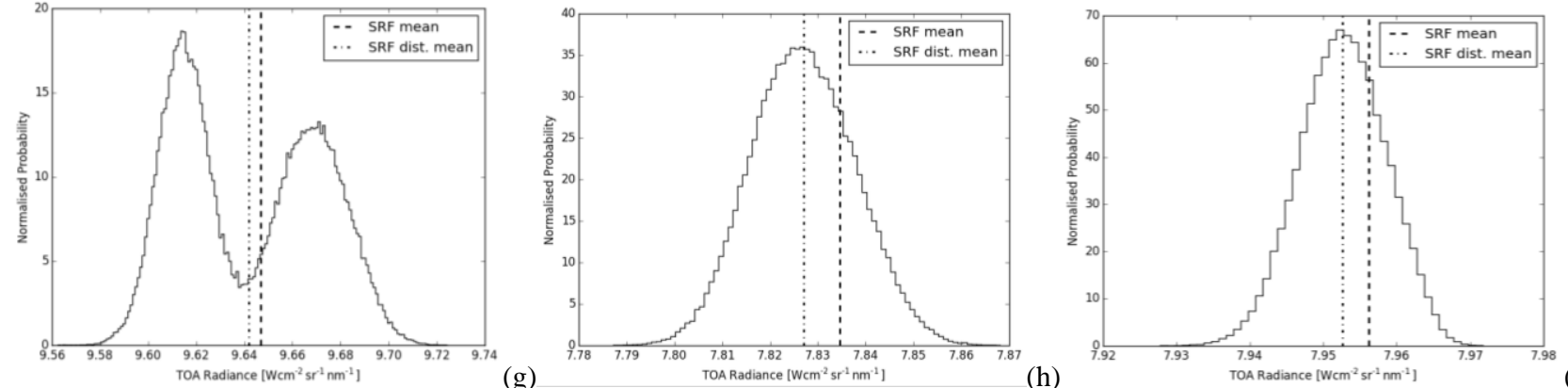

(f)

Figure 5. S2 spectral uncertainty results using cubic spline interpolation for B1 (a), B2 $(b)$ B3 $(c)$ B4 $(d)$ B5 $(e)$ B6 $(f)$ B7 $(g)$ $\mathrm{B} 8(h)$ and B8A $(i)$.

Table 1. Parameters associated to Figure 5.

\begin{tabular}{|c|c|c|c|c|c|c|c|c|c|}
\hline Parameter & B1 & B2 & B3 & B4 & B5 & B6 & B7 & B8 & B8A \\
\hline $\begin{array}{l}\text { Mean } \\
{\left[\mathrm{Wcm}^{-2} \mathrm{sr}^{-1} \mathrm{~nm}^{-1}\right]}\end{array}$ & 11.4227 & 11.2388 & 12.0806 & 10.8878 & 10.0079 & 10.1579 & 9.6419 & 7.8270 & 7.9527 \\
\hline $\mathrm{SD}[\%]$ & 0.22 & 0.01 & 0.01 & 0.04 & 0.05 & 0.21 & 0.31 & 0.14 & 0.07 \\
\hline $\begin{array}{l}\text { Median } \\
{\left[\mathrm{Wcm}^{-2} \mathrm{sr}^{-1} \mathrm{~nm}^{-1}\right]}\end{array}$ & 11.4241 & 11.2388 & 12.0807 & 10.8882 & 10.0079 & 10.1585 & 9.6393 & 7.8268 & 7.9527 \\
\hline Skewness & -0.3608 & 0.0461 & 0.3374 & -0.5002 & 0.0374 & -0.1917 & 0.1402 & 0.0766 & -0.0985 \\
\hline Kurtosis & 0.2939 & 0.0781 & 0.1876 & 0.0317 & 0.1261 & 0.0765 & -1.3022 & -0.2046 & -0.2157 \\
\hline $\begin{array}{l}\text { SRF mean } \\
{\left[\mathrm{Wcm}^{-2} \mathrm{sr}^{-1} \mathrm{~nm}^{-1}\right]}\end{array}$ & 11.4602 & 11.2385 & 12.0805 & 10.8876 & 10.0071 & 10.1650 & 9.6470 & 7.8345 & 7.9562 \\
\hline $\begin{array}{l}\text { Mean vs } \\
\text { SRF mean [\%] }\end{array}$ & -0.3310 & 0.0028 & 0.0012 & 0.0018 & 0.0085 & -0.0696 & -0.0524 & -0.0961 & -0.0446 \\
\hline
\end{tabular}



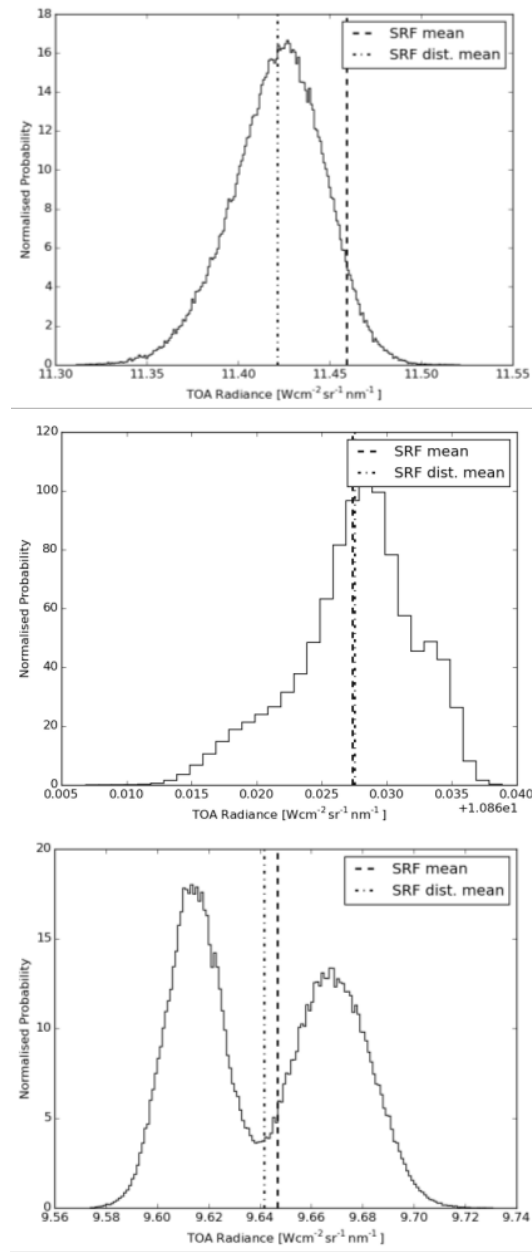

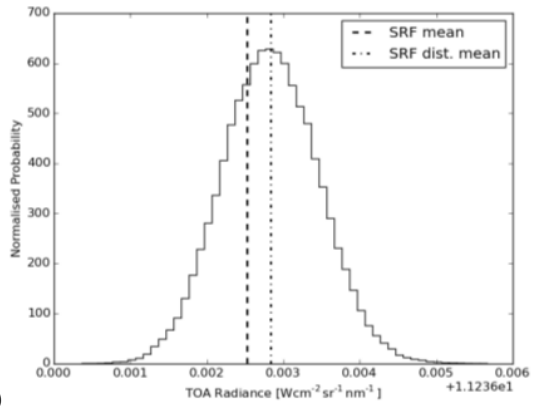

(a)
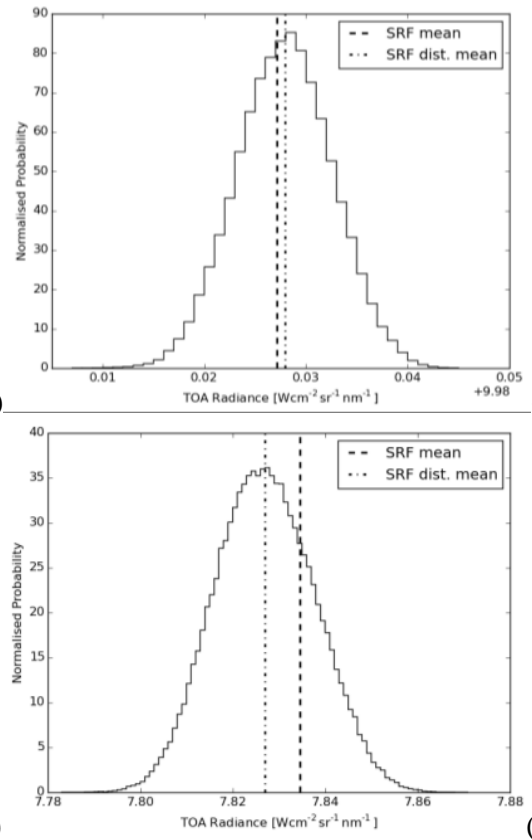

(b)

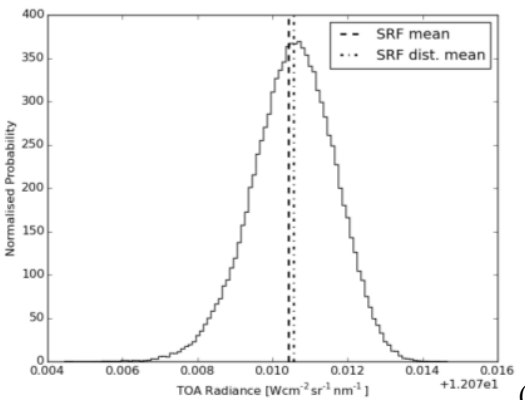

(c)

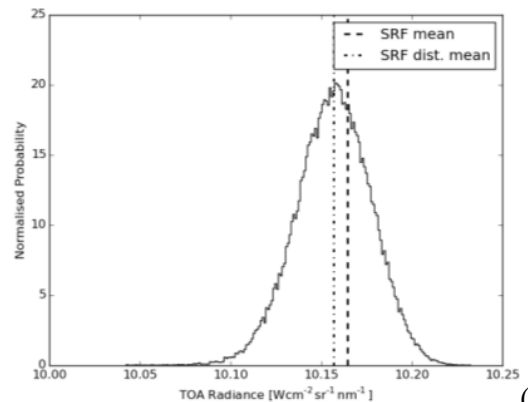

(f)

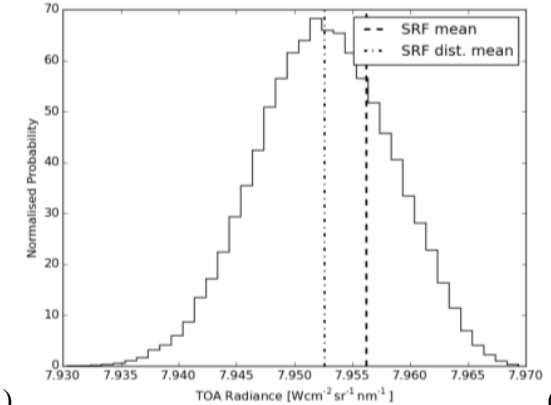

Figure 6. S2 spectral uncertainty results using PCHIP interpolation for B1 $(a)$, B2 $(b)$ B3 $(c)$ B4 $(d)$ B5 $(e)$ B6 $(f)$ B7 $(g)$ B8 (h) and B8A (i).

Table 2. Parameters associated to Figure 6.

\begin{tabular}{|c|c|c|c|c|c|c|c|c|c|}
\hline Parameter & B1 & $\overline{\text { B2 }}$ & $\overline{\text { B3 }}$ & B4 & B5 & B6 & B7 & B8 & B8A \\
\hline $\begin{array}{l}\text { Mean } \\
{\left[\mathrm{Wcm}^{-2} \mathrm{sr}^{-1} \mathrm{~nm}^{-1}\right]}\end{array}$ & 11.4215 & 11.2388 & 12.0806 & 10.8875 & 10.0079 & 10.1568 & 9.6416 & 7.8270 & 7.9526 \\
\hline $\mathrm{SD}[\%]$ & 0.22 & 0.01 & 0.01 & 0.04 & 0.05 & 0.20 & 0.31 & 0.14 & 0.07 \\
\hline $\begin{array}{l}\text { Median } \\
{\left[\mathrm{Wcm}^{-2} \mathrm{sr}^{-1} \mathrm{~nm}^{-1}\right]}\end{array}$ & 11.4228 & 11.2388 & 12.0806 & 10.8880 & 10.0079 & 10.1573 & 9.6392 & 7.8268 & 7.9526 \\
\hline Skewness & -0.2856 & 0.0825 & -0.2513 & -0.5251 & -0.0291 & -0.1943 & 0.1401 & 0.0662 & -0.0718 \\
\hline Kurtosis & 0.1342 & -0.0010 & 0.1543 & 0.0265 & -0.1299 & 0.2490 & -1.2985 & -0.2171 & -0.2778 \\
\hline $\begin{array}{l}\text { SRF mean } \\
{\left[\mathrm{Wcm}^{-2} \mathrm{sr}^{-1} \mathrm{~nm}^{-1}\right]}\end{array}$ & 11.4593 & 11.2385 & 12.0804 & 10.8873 & 10.0071 & 10.1645 & 9.6469 & 7.8345 & 7.9562 \\
\hline $\begin{array}{l}\text { Mean vs } \\
\text { SRF mean }[\%]\end{array}$ & -0.3310 & 0.0027 & 0.0011 & 0.0014 & 0.0079 & -0.0753 & -0.0546 & -0.0963 & -0.0450 \\
\hline
\end{tabular}


The results both using the PCHIP or cubic interpolation are quite consistent with minimum variations in the measured parameters. The difference in SD and error are minimum. The comparison of these two interpolation methods indicate the low effect of ill-conditioned interpolation situation. This is largely explained since the ill-conditions may appear for a small wavelength area as shown in Figure 4 but have a small effect in the integrated equivalent radiance $L_{\mathrm{eq}}$ in (1)

The SD is higher for B1 due to the high atmospheric contribution in that area combined with a $20 \mathrm{~nm}$ bandwidth. The B6 also introduces a relative higher SD due to its narrow bandwidth of only $15 \mathrm{~nm}$ and its strategic situation at the re-edge. Nonetheless, for a desert site this effect is minimized. The distribution shows also an important SD for B7 but, in this case, it is produced by a bi-modal distribution with approximately $0.6 \%$ difference mode-to-mode. That is the consequence of the filter manufacturing process. There are filter slices for each of the 12 detector modules that might be manufactured using different "wafers". This is a common situation with this type of push-broom instruments as occurs, for example, with Landsat-8 Operational Land Imager (OLI) ${ }^{15}$. The error introduced between the equivalent radiance $L_{\mathrm{eq}}$ and the mean of the distribution is generally negligible except for B1 when goes over $0.3 \%$. This is largely the consequence of a higher degradation rate and the narrow bandwidth of this band.

Therefore, for a cross-calibration over desert areas the uncertainty introduced by the spectral calibration knowledge must be accounted in the budget but the impact is expected to be relatively small compared to other uncertainty sources ${ }^{16}$. However, the systematic effects of non-uniformity and degradation might be considerable and minimized for specific bands. The spectral degradation of S2 is complicated to assess since there is no device on-board to track the spectral response during the mission life-time - as the Spectroradiometric Calibration Assembly (SRCA) on-board the Moderate Resolution Imaging Spectroradiometer (MODIS) mission ${ }^{8}$ — and the degradation rate becomes unpredictable. Tests with different degradation rates on $\mathrm{B} 1$ showed that the error increases with the degradation rate increase but also the SD as a result of changing the spectral region. This error is much lower for B2 since, although the degradation is similar to B1, its bandwidth is much larger. For the systematic effect introduced by the non-uniformity, the use of specific per-module SRF can introduce a substantial benefit in some bands as explained for B7 above.

For an operational integration of this uncertainty contribution, a look-up-table (LUT) could be generated by applying the same method to different TOA radiances. The example here considers a desert area with a typical application to the cross-calibration or instrument monitoring. It is expected that vegetation or coastal areas the SD values considerably increase for some bands. In addition, further work could include a more detailed representation of each one of the spectral uncertainty contributions to better understand the random and systematic effects. The spectral uncertainty should also include a certain knowledge on the SRF (not only the wavelength). In addition, further interpolation methods could be included. For this first exercise, the PCHIP and cubic spline interpolation were used. Others, as the Barycentric Lagrange interpolation as implemented in Figure 4 could be included in future iterations if a processing optimization is introduced by e.g. parallelization.

For the integration in the S2-RUT, the SD and error should be considered. The first one can be added in quadrature with the rest of the L1 contributors whereas the second one should be added linearly to the expanded uncertainty - the expanded uncertainty results from the multiplication of the standard uncertainty by the desired coverage factor $k$ - if no correction is introduced $^{3}$. The development of the S2-RUTv1 already includes this situation for some uncorrected effects as the diffuser degradation and the out-of-field stray-light effect- systematic part ${ }^{1}$.

\section{ORTHORECTIFICATION UNCERTAINTY PROPAGATION}

The first version of the S2-RUT provides the radiometric uncertainty at a pixel level but does not include the effect of the propagation over the radiometric resampling ${ }^{1}$. The effect of such a resampling will generally introduce a reduction of the uncertainty level due to the random components of the each pixel uncertainty in the resampling grid.

The first step in an orthorectification process is a geometric transformation that determines the position of the target point in the focal plane image. The target point in Sentinel-2 L1C product is the result of applying an Earth model in Universal Transverse Mercator (UTM) coordinates that includes a Digital Elevation Model (DEM). In order to determine its value, a radiometric interpolation between the focal plane points is performed. The process is illustrated in Figure 7. 


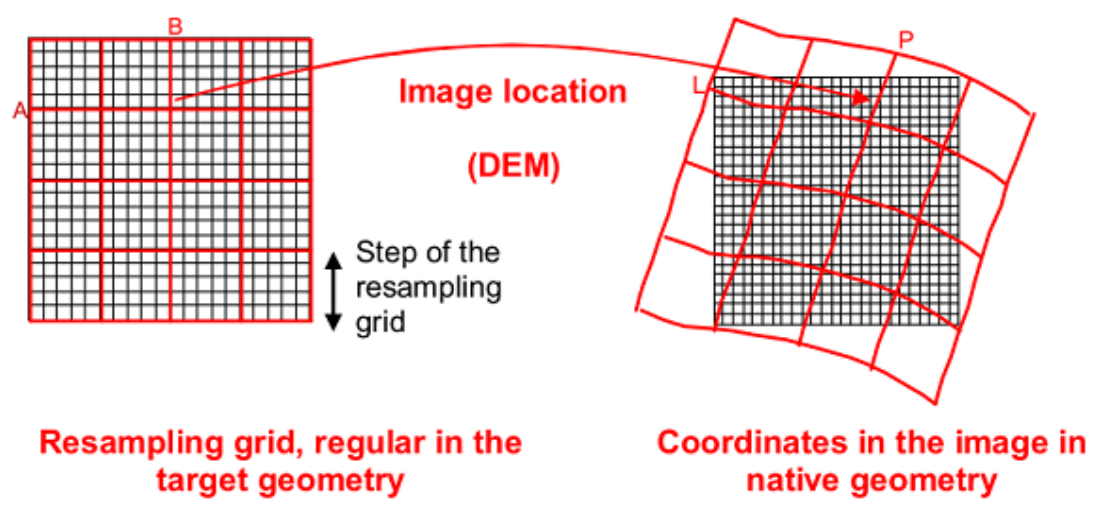

Figure 7. Resampling process of the S2 L1C products $^{17}$

In previous work, the combined standard uncertainty using the GUM was compared to the MCM approach to determine the validity of the combination ${ }^{18}$. The MCM approach models most of the L1B uncertainty contributions as either normal or rectangular distributions and propagates them through the L1B processing chain. The validation compares the $k=1$ uncertainty produced by the methods. Figure 8 shows the example for B6 at different radiance levels.
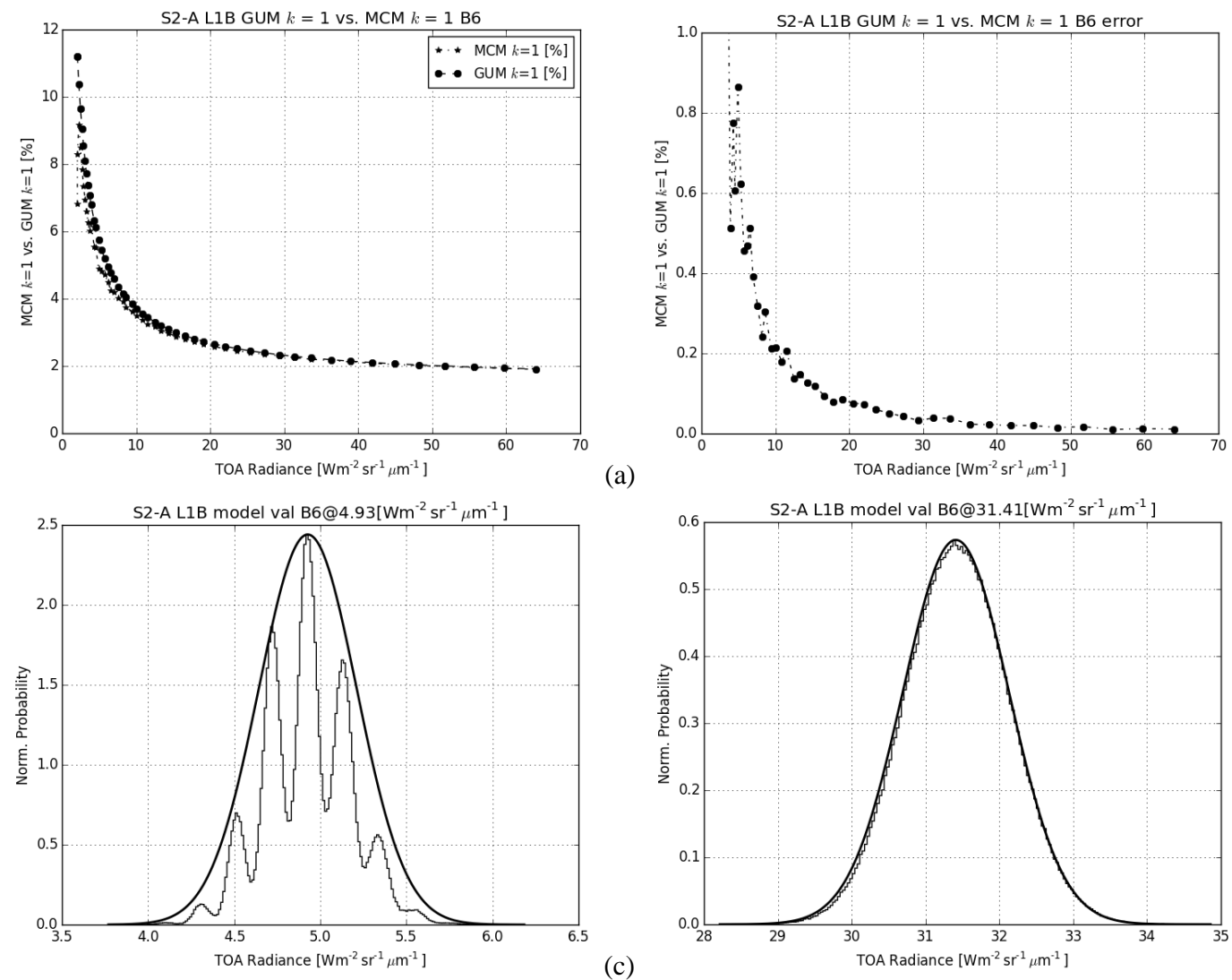

(a)

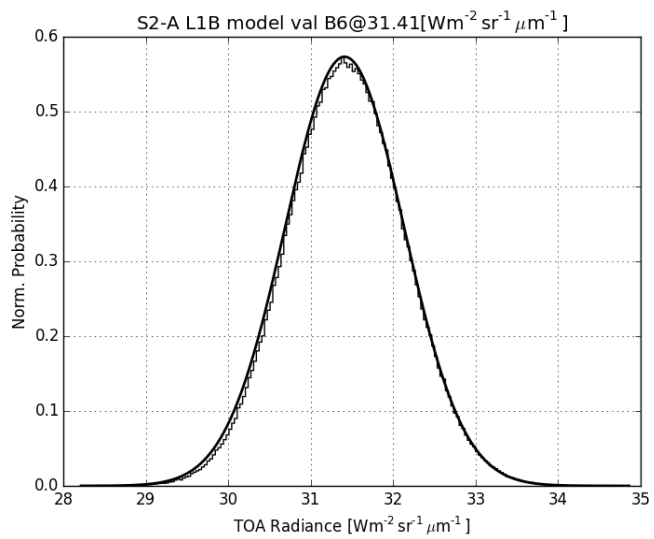

(b)

(c)

Figure 8. Results for the comparison of the GUM combined standard uncertainty and the MCM approach. The figure includes $(a)$ the uncertainty $k=1$ at several radiance levels up to the reference radiance ${ }^{2} L_{\text {ref }},(b)$ the error between the two approaches and the MCM distribution at (c) $4.93 \mathrm{Wm}^{-2} \mathrm{sr}^{-1} \mu \mathrm{m}^{-1}$ and $(d) 31.41 \mathrm{Wm}^{-2} \mathrm{sr}^{-1} \mu \mathrm{m}^{-1}$. Note that the distribution of the combined standard uncertainty has been re-scaled to the MCM distribution peak value.

The resulting major limitation for the uncertainty assessment was found to be produced by the Analog-to-Digital Converter (ADC) at the low radiances. At these low radiance values, the ADC quantization becomes unstable and cannot 
be modelled as a rectangular distribution anymore ${ }^{19}$. In that scenario, the central limit theorem cannot be applied and the combined standard uncertainty is not applicable ${ }^{3}$.

Selecting a resampling method is a complex decision that requires the evaluation of multiple criteria. Some of them are the processing time and the accuracy of the method. Here we describe a further criteria that describes the impact on the uncertainty propagation. That is, we want to study how the radiometric uncertainty of the focal plane measurements propagates through the different radiometric interpolations schemes down to a resampled image.

The nearest-neighbour method determines the resampled pixel by assigning the value of the closest pixel in the focal plane image. This method has low computational requirements and propagates without changes the uncertainty distribution from the focal plane to the resampled images. However, this comes at the expense of a low accuracy for nonuniform scenes.

Most of the resampling methods introduce a certain change in the radiance distribution when propagated from the focal plane to the resampled image. The implementation here propagates the resulting values of the MCM approach from the focal plane measurements to the resampled image by using three different interpolation methods. The first one is the bilinear method which uses a $2 \times 2$ grid. The second one is the cubic convolution. The method uses a cubic interpolation through the kernel that convolves with the original measurements ${ }^{20}$. The last method are the B-splines interpolation that is implemented as the nominal resampling method for the S2 L1C products ${ }^{17,21}$. Both cubic convolution and B-splines require a $4 \times 4$ kernel grid that produces a smoother image at the expense of a more demanding computational requirement ${ }^{20,21}$.

Figure 9 shows the results for the three different interpolation schemes presented above. The panels $(a),(b)$ and $(c)$ assume that the L1B uncertainty distribution are fully random and; thus, the samples in the grid are uncorrelated. The panels $(d),(e)$ and $(f)$ model the absolute calibration coefficient $A(b)$ as an uncertainty contributor which is fully correlated to all the L1B pixels in the grid. The results were obtained for a total of $20 \times 20$ positions in between the focal plane pixels for a kernel with a constant radiance of $30 \mathrm{Wm}^{-2} \mathrm{sr}^{-1} \mu \mathrm{m}^{-1}$.

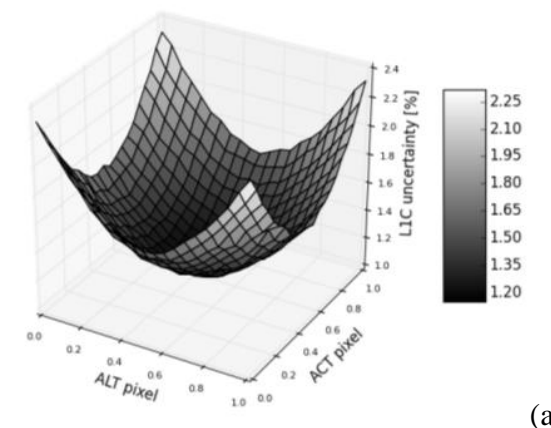

(a)

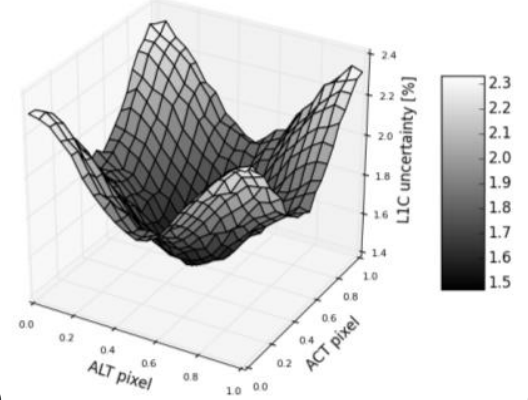

(b)

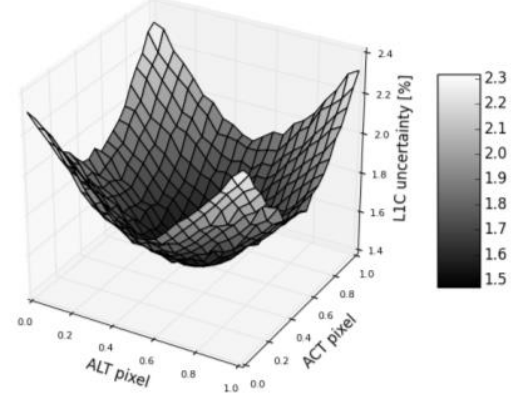

(c)

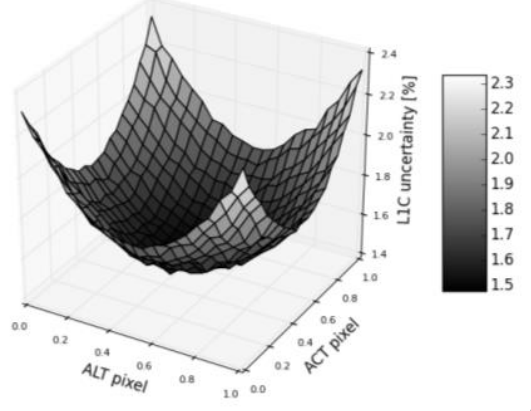

(d)

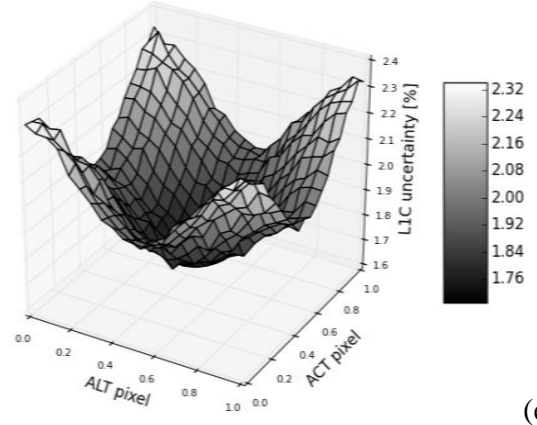

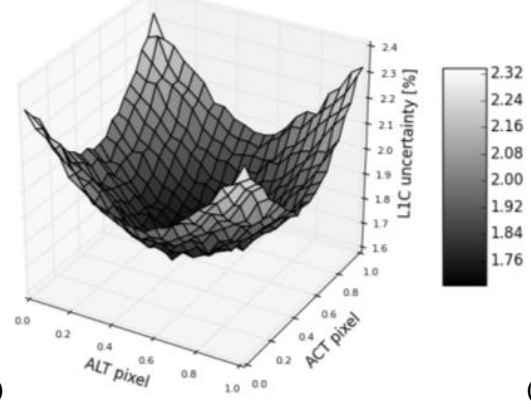

(f)

Figure 9. L1C uncertainty at different positions Across-Track (ACT) and Along-Track (ALT) of the pixel and full random L1B measurements using $(a)$ the bi-linear interpolation, $(b)$ cubic convolution and $(c)$ B-splines. L1C uncertainty at different positions of the pixel and introducing a fully correlated absolute calibration coefficient uncertainty $A(b)$ for the L1B measurements using $(d)$ the bi-linear interpolation, $(e)$ cubic convolution and $(f)$ B-splines. 
The results show the important variation on the propagated uncertainty depending on the collocation between the orthorectified and the focal plane grids. Table 3 summarizes the main parameters that describe the results of Figure 9.

Table 3. Parameters associated to the results in Figure 9.

\begin{tabular}{|l|l|l|l|l|l|l|}
\hline \multirow{2}{*}{ Parameter } & \multicolumn{2}{|c|}{ Uniform and full random } & \multicolumn{2}{|c|}{ Uniform and $\boldsymbol{A}(\boldsymbol{b})$ correlated } \\
\cline { 2 - 7 } & Bilinear & Cubic & B-spline & Bilinear & Cubic & B-spline \\
\hline Mean [\%] & 1.5631 & 1.8970 & 1.8141 & 1.7645 & 2.0012 & 1.9399 \\
\hline SD [\%] & 0.2603 & 0.2130 & 0.1943 & 0.1840 & 0.1593 & 0.1443 \\
\hline Minimum & 1.1491 & 1.4750 & 1.4718 & 1.4791 & 1.6891 & 1.6861 \\
\hline Maximum & 2.3165 & 2.3333 & 2.3165 & 2.3363 & 2.3420 & 2.3363 \\
\hline
\end{tabular}

The best results are obtained for the bi-linear interpolation. In all cases, it obtains the lowest mean and minimum values. This interpolation does provide an improved randomisation at the expense of a low "smoothness". The B-spline obtains a better mean value for the $20 \times 20$ positions than the cubic convolution. Although the minimum value for the two methods is quite close, the distance in the mean is slightly larger. This is produced since the cubic convolution introduces a nonstable interpolation close to the kernel points that introduce slightly higher uncertainty than the focal plane measurements — see the maximum is $2.3333 \%$ compared to $2.3165 \%$ for the rest of the cases.

The differences between the fully uncorrelated kernel points with the case where $A(b)$ is fully correlated demonstrates the importance of the correlation in the uncertainty propagation. Table 3 reports differences in the mean value of approximately $0.1 \%$ for the cubic convolution and B-spline interpolation and as large as $0.2 \%$ for the bi-linear interpolation.

For its implementation in future versions of the S2-RUT, the uncertainty estimates at the L1C - orthorectified images will be limited to assess the position in the grid and; thus the effect of the uncertainty propagation. A simple implementation would introduce a "reduction factor" of the L1B uncertainty estimates based on an analysis as above. That "reduction factor" would be based in an analysis as the one demonstrated above that compares the kernel uncertainty estimates with the potential uncertainty at different positions on the grid.

The selected approach will largely depend on the user requirements and the application. That is, if the user application involves the a-posteriori binning of the pixels, a large fraction of the uncorrelated uncertainty contributions will be reduced. In that case, the resampling uncertainty propagation will have a smaller impact to the user.

The best implementation of the uncertainty propagation would imply the assessment of the uncertainty at the L1B products and the propagation through the resampling by using pre-calculated Look-Up-Tables (LUT). Similarly to the method presented above, the LUTs can be populated for several radiance values in the grid and positions in the pixel. However, for a kernel of $4 \times 4$ L1B radiance values, the number of cases could be sensibly high and a minimization of cases should be pursued.

Figure 10 and Table 4 show the differences in the interpolated values between the different resampling methods for a case where the radiance kernel is non-uniform. As previously commented, not only the resampling introduces a change in the uncertainty distribution but also includes an accuracy of the resampled value. That is, how well the interpolation represents the "real" scene variation. For the uniform scene previously studied, the three interpolation obviously have a perfect accuracy. In that case, a bi-linear interpolation requires less processing and can potentially better reduce the radiometric uncertainty. However, most of the scenes will not be close or near-close to a uniform TOA radiance.

The Figure 10 shows the large differences that can be obtained by using different resampling methods. In that case it is difficult to determine which the best method is since assumptions of the scene inter-pixel variations are needed. The Table 4 quantifies these differences by calculating the root mean squared error (RMSE) between the different interpolation methods. The RMSE is considerably high between any of the three methods. If a radiance of $30 \mathrm{Wm}^{-2} \mathrm{sr}^{-}$ ${ }^{1} \mu \mathrm{m}^{-1}$ is taken as reference, the RMSE is approximately $5 \%$ between the bi-linear method and both the cubic convolution and B-spline whereas the difference between the cubic convolution and B-spline is as large as $2 \%$. 
However, it can be appreciated in Figure 10 how these differences are not constant but can be much higher or lower depending on the position on the grid. For example, it can be seen how the area close to $(0,0)$ is more unstable than the area close to $(1,1)$ in panel $(b)$.

Table 4. RMSE error between the interpolated radiance of panels $(a),(b)$ and $(c)$ from Figure 10

\begin{tabular}{|l|l|l|l|}
\hline RMSE $\left[\mathbf{W m}^{-2} \mathbf{s r}^{-1} \boldsymbol{\mu m ^ { - 1 }}\right]$ & Bilinear & Cubic & B-spline \\
\hline Bilinear & 0.0000 & 1.6773 & 1.3559 \\
\hline Cubic & 1.6773 & 0.0000 & 0.6969 \\
\hline B-spline & 1.3559 & 0.6969 & 0.0000 \\
\hline
\end{tabular}

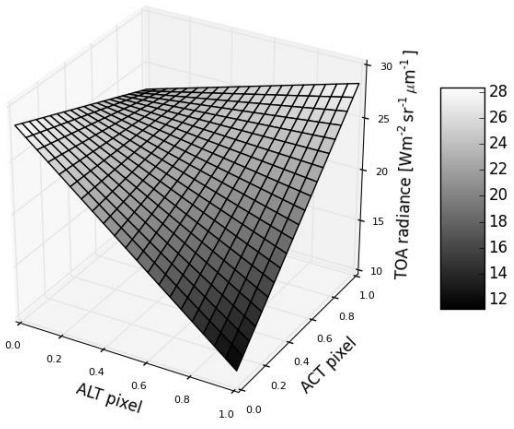

(a)

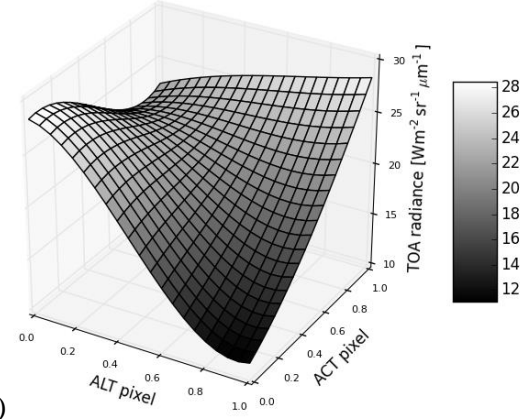

(b)

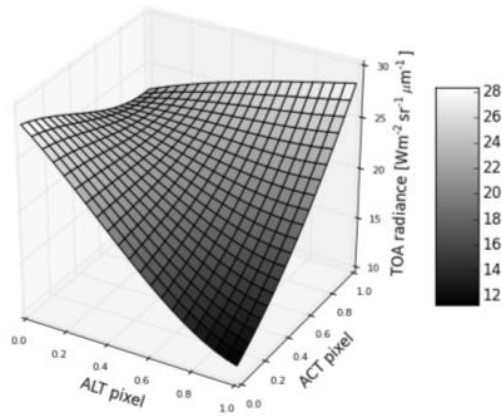

(c)

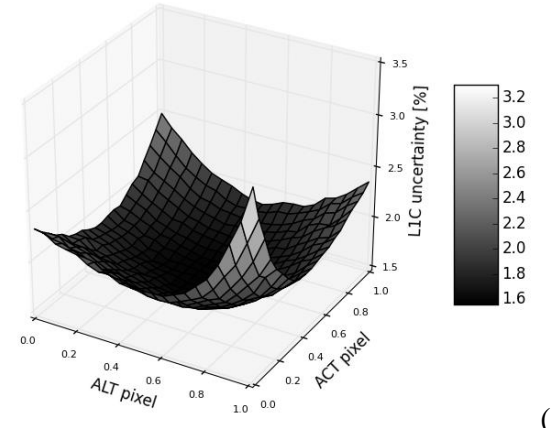

(d)

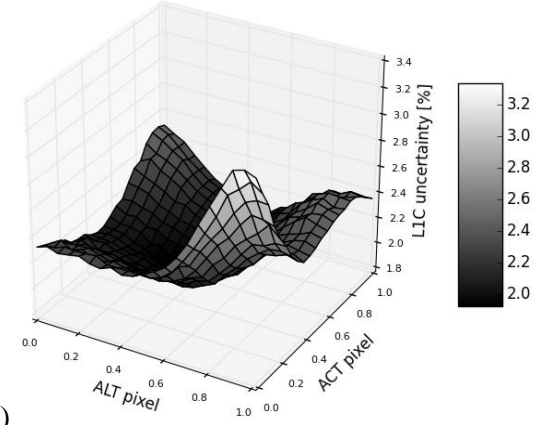

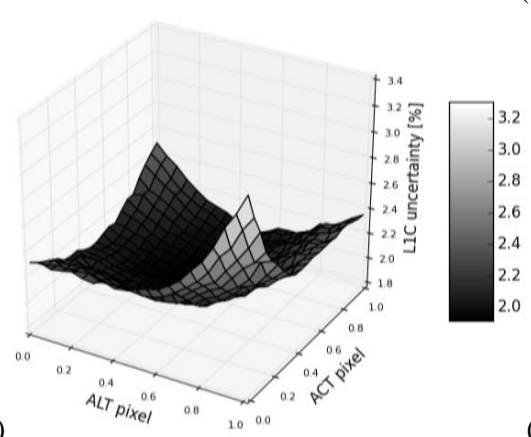

(f)

Figure 10. L1C radiance at different positions Across-Track (ACT) and Along-Track (ALT) of the pixel and introducing a fully correlated absolute calibration coefficient uncertainty $\mathrm{A}(\mathrm{b})$ for the L1B measurements using the bi-linear interpolation (a), cubic convolution (b) and B-splines (c). L1C uncertainty at different positions of the pixel and introducing a fully correlated absolute calibration coefficient uncertainty $A(b)$ for the L1B measurements using the bi-linear interpolation $(d)$, cubic convolution $(e)$ and B-splines $(f)$. Non-uniform radiance kernel (values from left to right and up to down in a $4 \times 4$ kernel) [[21, 20, 10, 14], [15, 30, 24, 50], [10, 12, 30, 60], [12, 45, 36, 15]] $\mathrm{Wm}^{-2} \mathrm{sr}^{-1} \mu \mathrm{m}^{-1}$.

This is a first implementation of a complex topic in the estimation of radiometric uncertainty. Further work could include the impact of the knowledge on the geometric transformation and how this position grid knowledge influences the interpolated radiance as well as the propagated uncertainty. In addition, the resampling can be performed using a predetermined gridding. In that case, the gridding precision should be also assessed. Finally, the effect of the resampling at the image boundaries e.g. by mirroring should be carefully analyzed.

\section{CONCLUSIONS AND FURTHER WORK}

The work here describes the research of novel methods to describe the spectral response uncertainty and the orthorectification uncertainty propagation.

The spectral response module is capable of determining the effect of the spectral calibration knowledge, spectral nonuniformity and spectral degradation in an integrated software. The spectral calibration knowledge has been further 
separated into a random and systematic component and the components propagated using a MCM approach. The example used here represents a desert TOA radiance that could be typically used for instrument cross-calibration. In that case, the uncertainty introduced by the spectral calibration knowledge must be accounted in the budget but the impact is expected to be relatively small compared to other uncertainty sources ${ }^{16}$. The degradation effect has shown a considerable error on B1 For the systematic effect of the non-uniformity, the use of specific per-module SRF can produce a substantial benefit in certain bands as, for example, B7.

The orthorectification module propagates the L1B uncertainty up to the L1C using a MCM approach in a kernel of $4 \times 4$ L1B radiance values for the cubic convolution and B-spline interpolation $(2 \times 2$ kernel for the bi-linear case). The results show the important variation on the propagated uncertainty depending on the collocation between the orthorectified and the focal plane grids. The results also show how the bi-linear interpolation effectively better reduces the radiometric uncertainty at L1C. The uncertainty reduction rate largely depends on the level of correlation between the L1B kernel points. For non-uniform scenes, the bi-linear method still better reduces the L1C uncertainty but important differences in the interpolated radiance between the three methods were found. To fully account the effect of the orthorectification, the estimation should be assessed take into account the L1B product. However, if that is not possible, a rough estimation can be applied using the L1C products as an input.

Further work is needed for a full assessment and the integration of these uncertainty contributions in future versions of the S2-RUT tool. The example in this paper primarily considers a desert area with a typical application to the crosscalibration or instrument monitoring. For an operational integration, a look-up-table (LUT) could be generated for both uncertainty contributions by applying the same method to different TOA radiance scenes. The integration of both uncertainty contributors in the S2-RUT uncertainty combination should considered both the uncertainty and the error. As previously commented, the first one can be added in quadrature with the rest of the L1 contributors whereas the second one should be added linearly to the expanded uncertainty if no correction is introduced ${ }^{3}$.

The spectral uncertainty module could include a detailed representation of each one of the spectral calibration uncertainty contributions and other interpolation methods as the Barycentric Lagrange one. The spectral uncertainty could also include a certain knowledge on the SRF (not only the wavelength) and a detailed analysis of the potential degradation scenarios.

The orthorectification module improvement should be in an in-depth analysis of the covariance between the L1B pixels. This would determine the level of uncertainty improvement at the L1C pixels. In addition, further work must follow in the identification of the potential accuracy error introduced by the interpolation method.

\section{REFERENCES}

[1] J. Gorroño, N. Fomferra, and M. Peters, "Sentinel-2 Radiometric uncertainty tool (S2-RUT)," European Space Agency, 10 May 2016, https://github.com/senbox-org/snap-rut (23 August 2016)

[2] M. Drusch, U. Del Bello, S. Carlier et al., "Sentinel-2: ESA's Optical High-Resolution Mission for GMES Operational Services," Remote Sensing of Environment, 120, 25-36 (2012).

[3] BIPM, IEC, IFCC et al.,"Guide to the Expression of Uncertainty in Measurement," JCGM 100:2008, (2008). Available at: http://www.bipm.org/utils/common/documents/jcgm/JCGM_100_2008_E.pdf.

[4] BIPM, IEC, IFCC et al.,"Supplement 1 to the 'Guide to the Expression of Uncertainty in Measurement'Propagation of distributions using a Monte Carlo method," JCGM 101:2008, (2008). Available at: http://www.bipm.org/utils/common/documents/jcgm/JCGM_101_2008_E.pdf.

[5] J. L. Gardner, "Uncertainties in Interpolated Spectral Data,” J. Res.Natl. Inst. Stand. Technol., 108(1), 69-78 (2003).

[6] S. Clerc,"S2 MPC: Data Quality Report," 21 July 2016. Available at: https://sentinel.esa.int/web/sentinel/dataproduct-quality-reports.

[7] A. Berk, G. P. Anderson, P. K. Acharya et al., "MODTRAN5: a reformulated atmospheric band model with auxiliary species and practical multiple scattering options." 5425, 341-347.

[8] X. Xiong, N. Che, and W. Barnes, "Terra MODIS on-orbit spectral characterization and performance," Geoscience and Remote Sensing, IEEE Transactions on, 44(8), 2198-2206 (2006).

[9] H. Takashashi, "Temperature stability of thin-film narrow-bandpass filters produced by ion-assisted deposition," Applied Optics, 34(4), 667-675 (1995). 
[10] P. Dierckx, [Curve and surface fitting with splines] Oxford University Press, Inc., (1993).

[11] F. N. Fritsch, and R. E. Carlson, "Monotone Piecewise Cubic Interpolation," SIAM Journal on Numerical Analysis, 17(2), 238-246 (1980).

[12] J.-P. Berrut, and L. N. Trefethen, "Barycentric Lagrange Interpolation,” SIAM Review, 46(3), 501-517 (2004).

[13] J. Gorroño, A. Bialek, P. D. Green et al., "Non-normal distribution of the top-of-atmosphere satellite optical measurements over calibration sites," International Journal of Remote Sensing, 37(19), 4665-4682 (2016).

[14] D. Zwillinger, and S. Kokoska, [CRC Standard Probability and Statistics Tables and Formulae] CRC Press (1999).

[15] J. Barsi, K. Lee, G. Kvaran et al., "The Spectral Response of the Landsat-8 Operational Land Imager," Remote Sensing, 6(10), 10232 (2014).

[16] G. Chander, D. L. Helder, D. Aaron et al., "Assessment of Spectral, Misregistration, and Spatial Uncertainties Inherent in the Cross-Calibration Study," IEEE Transactions on Geoscience and Remote Sensing, 51(3), 12821296 (2013).

[17] ESA, "Sentinel-2 MSI Technical Guide," European Space Agency, https://sentinel.esa.int/web/sentinel/technical-guides/sentinel-2-msi (12 July 2016)

[18] J. Gorroño, F. Gascon, and N. P. Fox, "Radiometric uncertainty per pixel for the Sentinel-2 L1C products." 96391G.

[19] P. Carbone, and D. Petri, "Mean value and variance of noisy quantized data," Measurement, 23(3), 131-144 (1998).

[20] R. Keys, “Cubic convolution interpolation for digital image processing," IEEE Transactions on Acoustics, Speech, and Signal Processing, 29(6), 1153-1160 (1981).

[21] H. Hsieh, and H. Andrews, "Cubic splines for image interpolation and digital filtering," IEEE Transactions on Acoustics, Speech, and Signal Processing, 26(6), 508-517 (1978). 